\title{
Seed-Relative Segmentation Robustness of Watershed and Fuzzy Connectedness Approaches
}

\author{
Romaric Audigier, Roberto Lotufo \\ School of Electrical and Computer Engineering \\ State University of Campinas - UNICAMP \\ C.P. 6101, 13083-852, Campinas (SP), Brazil \\ audigier@dca.fee.unicamp.br, www.dca.fee.unicamp.br/ lotufo
}

\begin{abstract}
This paper analyzes the robustness issue in three segmentation approaches: the iterative relative fuzzy object extraction, the watershed transforms (WT) by image foresting transform and by minimum spanning forest. These methods need input seeds, which can be source of variability in the segmentation result. So, the robustness of these segmentation methods in relation to the input seeds is focused. The core of each seed is defined as the region where the seed can be moved without altering the segmentation result. We demonstrate that the core is identical for the three methods providing that the tie-zone transform has previously been applied on these methods. Indeed, as the two WT approaches do not return unique solution, the set of possible solutions has to be considered in a unified solution. So does the tie-zone transform. As opposed to what we could think, we show that the core is included in but different from the catchment basin. We also demonstrate that the tie-zone transforms of these WTs are always identical. Furthermore, the framework of minimal sets of seeds, an inverse problem of segmentation, is extended to the pixel level and related to the cores. A new algorithm for the computation of minimal seed sets is finally proposed.
\end{abstract}

\section{Introduction}

Watershed transform [5] and fuzzy connectedness [13] are successful approaches to segment an image. Both need seeds on the input to define the segments of interest. Seeds can be either picked by the user or automatically selected. The choice of seeds is however a source of variability. Indeed, manual choice can vary depending on the user and the moment. Automatic selection of seeds also depends of filtering or other processing which generally depends on parameters. It is why we study in this paper the theoretical problem of segmentation robustness in relation to the input seeds: the characterization of the cores. The core of each seed is defined as the region where the seed can be moved without altering the segmentation result. As opposed to what we could think, the core is different from the catchment basin. We also analyze the inverse problem of segmentation, i.e., the possible sets of seeds that correspond to a target segmentation: the so-called minimum sets of seeds.

Three segmentation methods are focused: the iterative relative fuzzy object extraction (IRFOE) [12], the watershed transform by image foresting transform (IFT-WT) [6] and the watershed transform by minimum spanning forest (MSF-WT) [11]. As the two WT approaches do not return unique solution, which is contrary to the idea of robustness, we use their respective tie-zone transforms [3] (TZ-IFT-WT and TZ-MSF-WT) which unify the set of possible solutions.

This paper has many theoretical contributions: a general characterization of the cores for the three segmentation methods, the demonstration of equivalence between TZIFT-WT and TZ-MSF-WT methods, the relationship between cores and non-redundant receptive regions under the framework of minimal seed sets, and the extension of minimal seed sets at pixel level with a new algorithm for their computation.

The organization of the paper is as follows. We recall the basic definitions of the three segmentation methods based on watershed and fuzzy connectedness in Section 2. Section 3 deals with robustness problem: Core definitions for IRFOE and TZ-IFT-WT are recalled in Section 3.1. Section 3.2 demonstrates that the sets of shortest-path forests with max-arc path cost and minimum spanning forests give identical segmentation, then shows that the core is identical for the three methods (IRFOE, TZ-IFT-WT and TZ-MSFWT). Section 4 recalls the minimal seed set framework, extends it at pixel level, relates it to cores and presents the new algorithm. 
Table 1. Table of abbreviations

\begin{tabular}{ll}
\hline IRFOE & iterative relative fuzzy object extraction \\
WT & watershed transform \\
IFT & image foresting transform \\
SPF & shortest path forest \\
SPF-max & shortest path forest \\
& using the max-arc path cost \\
MSF & minimum spanning forest \\
TZ & tie zone \\
MSS & minimal seed set \\
NRRR & non-redundant receptive region \\
\hline
\end{tabular}

\section{Seeded segmentation methods}

In this section, we briefly recall the basic definitions of the three segmentation paradigms focused in the paper: the watershed transform by image foresting transform (IFTWT), the watershed transform by minimum spanning forest (MSF-WT), and the iterative relative fuzzy object extraction (IRFOE).

\subsection{Watershed by image foresting transform}

The watershed transform (WT) is a famous and powerful segmentation tool in morphological image processing, first introduced by Beucher and Lantuéjoul [4] for contour detection and applied in digital image segmentation by Beucher and Meyer [5].

Under the image foresting transform (IFT) framework [6], the watershed transform is viewed as a graph optimization problem: creation of a shortest-path forest (SPF).

An image is interpreted as a weighted graph $G=$ $(V, A, w)$ consisting of a set $V$ of nodes representing the image pixels, a set $A$ of arcs weighted by $w$, a function from $A$ to some nonnegative scalar domain. $N(v)$ denotes the neighborhood of node $v$, i.e. the set of nodes adjacent to it. Nodes $u$ and $v$ are adjacent when the arc $\langle u, v\rangle$ belongs to $A$. A graph $\left(V^{\prime}, A^{\prime}\right)$ is subgraph of $(V, A)$ if $V^{\prime} \subseteq V$, $A^{\prime} \subseteq A$ and $A^{\prime} \subseteq V^{\prime} \times V^{\prime}$. A forest $F$ of $G$ is an acyclic subgraph $F$ of $G$. Trees are connected components of the forest (any two nodes of a tree are connected by a path). A path $\pi(u, v)$ from node $u$ to node $v$ in graph $(V, A, w)$ is a sequence $\left\langle u=v_{1}, v_{2}, \ldots, v_{n}=v\right\rangle$ of nodes of $V$ such that $\forall i=1 \ldots n-1,\left\langle v_{i}, v_{i+1}\right\rangle \in A$. A path is said simple if all its nodes are different from each other. The path is trivial when it consists of a single node $\langle v\rangle$. A path-cost function $f$ assigns to each path $\pi$ a path cost $f(\pi)$, in some totally ordered set of cost values.

Let $S \subseteq V$ be a set of particular nodes $s_{i}$ called seeds. For a given weighted graph $(V, A, w)$ and a set $S$ of seeds, the image foresting transform (IFT) returns a forest $S P F$ of $(V, A, w)$ such that (i) there exists for each node $v \in V$ a unique and simple path $\pi\left(s_{i}, v\right)$ in $S P F$ from a seed node $s_{i} \in S$ to $v$ and (ii) each such path is optimum, i.e., has a minimum cost for linking $v$ to some seed of $S$, according to the specified path-cost function $f$.

The watershed transform by IFT (IFT-WT) assumes that the max-arc path-cost function $f_{\max }$ is used:

$$
\begin{aligned}
f_{\max }\left(\left\langle v_{1}\right\rangle\right) & =0 \\
f_{\max }\left(\left\langle v_{1}, v_{2}, \ldots, v_{n}\right\rangle\right) & =\max _{i=1, \ldots, n-1}\left\{w\left(v_{i}, v_{i+1}\right)\right\}
\end{aligned}
$$

where $w(u, v)$ is the symmetric weight of arc $\langle u, v\rangle \in A$, ideally higher on the object boundaries and lower inside the objects.

Usual arc weight functions are: $w_{1}(u, v)=\mid I(u)-$ $I(v) \mid, I(u)$ being the intensity of pixel $u$; $w_{2}(u, v)=$ $\max \{G(u), G(v)\}$, where $G(u)$ is the (morphological) gradient of image $I$ at pixel $u$.

A label map $L$ assigns to each node $v$ the label $L(v)$ of the corresponding minimum-path root. The catchment basins correspond to the (labeled) trees: $C B_{I F T}\left(s_{i}\right)=$ $\left\{v \in V, L(v)=L\left(s_{i}\right)\right\}$. Note that the final cost map $C$ is unique and corresponds to the morphological superior reconstruction of the image from the seeds using a flat structuring element. However, the forests and then the labelings may be multiple.

Instead of choosing one of the many labelings, the tiezone concept [3] can be applied to unify the multiple solutions of a WT: Regions where possible labelings differ are put in the tie zone (TZ). In the case of IFT-WT, the tie-zone watershed by IFT (TZ-IFT-WT), returns a unique partition of the image made of catchment basins and tie zone:

$$
\begin{aligned}
C B_{T Z-I F T}\left(s_{i}\right) & =\left\{v \in V, \forall F \in \Phi, \exists \pi\left(s_{i}, v\right) \text { in } F\right\} \\
T Z_{I F T} & =V \backslash \bigcup_{i} C B_{T Z-I F T}\left(s_{i}\right)
\end{aligned}
$$

where $\Phi$ denotes the set of the shortest-path forests $F$ (IFTs).

\subsection{Watershed by minimum spanning forest}

Another watershed transform can be defined from the minimum spanning forest. A minimum spanning forest (MSF) for graph $(V, A, w)$ is a forest $\left(V, A^{*}\right)$ whose total weight $\sum_{a \in A^{*}} w(a)$ (sum of the weights of its arcs) is minimum and where each node $v \in V$ is linked to a seed $s_{i} \in S$ by a unique simple path. The watershed transform by minimum spanning forest (MSF-WT) introduced in [10] is applied on a weighted neighborhood graph whose nodes are the primitive catchment basins corresponding to regional 


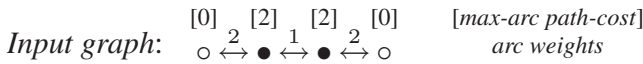

$$
\begin{aligned}
& \mathrm{A} \stackrel{2}{\leftrightarrow} \mathrm{A} \stackrel{1}{\leftrightarrow} \mathrm{A} \quad \mathrm{B} \quad \mathrm{A} \quad \mathrm{B} \stackrel{1}{\leftrightarrow} \mathrm{B} \stackrel{2}{\leftrightarrow} \mathrm{B} \quad \mathrm{A} \stackrel{2}{\leftrightarrow} \mathrm{A} \quad \mathrm{B} \stackrel{2}{\leftrightarrow} \mathrm{B}
\end{aligned}
$$

$\begin{array}{lll}\text { (a) MSF } 1 \text { and SPF } 1 & \text { (b) MSF } 2 \text { and SPF } 2 & \text { (c) SPF } 3 \text { is not a MSF }\end{array}$

\section{Figure 1. A weighted graph with two seeds (0)} and its 3 possible SPF-max and 2 MSFs.

minima of the image, and whose arcs link neighbor catchment basins and are weighted by the altitude of the pass between them. In fact, the same paradigm can be applied on graph where nodes are pixels and arcs have higher weight between dissimilar nodes (like a gradient). The many possible MSFs on a weighted graph define partitions that are solutions of this WT: Each tree of the MSF is a catchment basin of the MSF-WT, and nodes can be labeled in accordance with their seed label. Tie-zone transform can also be applied to unify the multiple solutions and get a unique labeling.

For a given weighted graph, the set of MSF-WT solutions is a subset of the set of IFT-WT solutions using the max-arc path cost.

Proposition 1 ([2]) Any minimum spanning forest (MSF) is also a shortest-path forest (SPF-max) using max-arc path cost $f_{\max }$.

Reciprocal is false as illustrated in Fig. 1: Three SPFmax can be built from a weighted graph and its two seeds $A$ and $B$ (represented by $\circ$ ). Cutting any arc results in a SPF-max. Yet, we must cut one of the arcs of weight 2 to obtain MSFs of total weight 3 (cf. Fig. 1(a)(b)).

\subsection{Fuzzy connectedness approach}

Many approaches of segmentation by fuzzy connectedness have been proposed. We focus on one of them: the iterative relative fuzzy object extraction (IRFOE) [12]. It is shown in ref. [1] that this approach is the dual of the TZIFT-WT: Fuzzy affinity $\mu_{\kappa}(u, v)$ (similarity measure) between nodes $u$ and $v$ plays the role of arc weight $w(u, v)$; path strength defined as the minimum affinity in the path corresponds to the max-arc path cost; and the strength of fuzzy connectedness $\mu_{K}(s, u)$ between two nodes (maximum strength of the paths between them) is the dual of the optimal (minimum) path cost. The subsequent robustness results developed in the IFT-WT framework will therefore be valid for the IRFOE approach too.

Observe that this approach uses a unique symmetric affinity function. If multiple asymmetric affinity functions are used as in ref. [8], the duality with TZ-IFT-WT is no longer valid. Such a segmentation does not respect any global optimality criterion, but is similar to a flooding process where optimization is only achieved temporarily at each flooding level but not necessarily at the end of the segmentation process. Although it is possible to define cores for robustness of such a paradigm, we will not deal with it because more complex and longer mathematical treatment would be necessary.

\section{Robustness regions for seeds}

Now the three segmentation methods of interest have been presented, the seed-relative robustness issue can be dealt with. The problem addressed in refs. $[12,1]$ is to find the cores, i.e., the regions where seeds can be moved without altering the segmentation (or labeling). In other words, the spans of the trees must be the same after moving the seeds within the cores (trees may change). Figure 2 illustrates the concepts of catchment basins, tie zone, cores of seeds, for a $4 \times 8$ pixel image. Figure 2 (c) shows the cores (in gray and white) where the seeds (represented by diamonds) may be displaced without altering the segmentation of Fig. 2(b), i.e., a partition of the image in two catchment basins (in gray and white) and a tie zone (in black).

In the next subsections, we recall the theoretical characterization $[12,1]$ of the core in the case of IFT-WT and its dual IRFOE, then, extend it to the case of MSF-WT.

\subsection{Cores for the shortest-path forests and the iter- ative relative fuzzy object}

In the case of IRFOE, the core relative to a seed corresponds to its relative fuzzy object (RFO) [12], i.e., the set of pixels that have strictly higher strength of connectedness with this seed than with any other seed:

$$
K_{s}=\left\{v \in V, \mu_{K}(s, v)>\mu_{K}(t, v), \forall t \in S, t \neq s\right\}
$$

The robustness property of the core was thoroughly demonstrated in ref. [12].

By duality, the core relative to a seed for the TZ-IFT-WT was also defined [1]: it is the set of pixels that have at least a path from this seed with strictly lower cost than any path from other seeds. As the cost of the minimum path from a seed $s \in S$ to any pixel $v$ can be given by the superior reconstruction $C_{s}[v]$ of the image from this seed, the core $K_{s}$ relative to a seed $s$ is defined by:

$$
K_{s}=\left\{v \in V, \quad C_{s}[v]<C_{t}[v], \quad \forall t \in S, \quad t \neq s\right\}
$$

Note that the pixels with paths of same (non-strict) minimum cost linking to different seeds do not belong to any core. They can belong either to the tie-zone (if these paths are optimal, i.e., for all path nodes, the cost is minimum, 


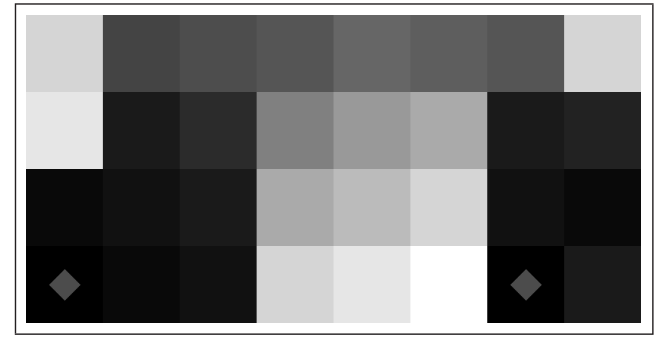

(a)

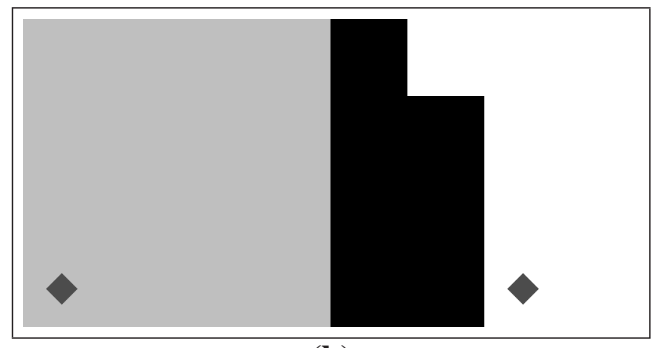

(b)

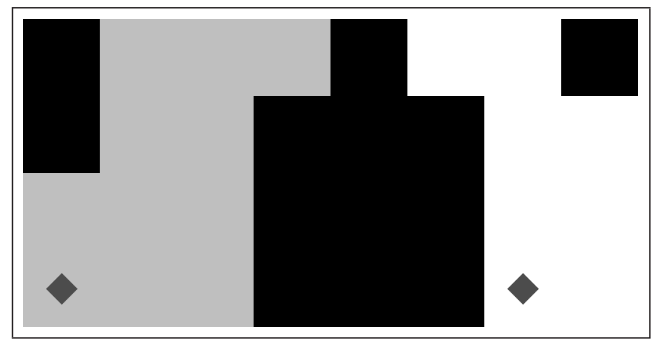

(c)

Figure 2. (a) Gray-level gradient image and seeds (diamonds). (b) Catchment basins (gray and white) and tie-zone (black). (c) Cores (gray and white) of the seeds (diamonds).

not only for the final node); or to the protected zone (complement of the core in a catchment basin) of a catchment basin.

An equivalent definition of the core uses the pass-value concept. The pass-value of a path is equal to its highest arc: $\epsilon(\pi)=\max _{\langle p, q\rangle}$ in $\pi\{w(p, q)\}=f_{\max }(\pi)$. The pass-value between two nodes $p$ and $q$ is equal to the minimum pass-value of the paths between them: $\epsilon(p, q)=$ $\min _{\forall \pi: p \rightsquigarrow q}\{\epsilon(\pi)\}=C_{p}[q]$. The pass-value of a seed $s$ is equal to the minimum of the pass-values between $s$ and any other seed: $\epsilon(s)=\min _{\forall t \in S, t \neq s}\{\epsilon(s, t)\}$. The core is then defined by:

$$
K_{s}=\{v \in V, \quad \epsilon(s, v)<\epsilon(s)\}
$$

\subsection{Cores for the minimum spanning forests}

As opposed to what one could think (p. 81, [10]), the catchment basins defined by the MSF-WT are not robustness regions. Figure 3 shows a counter-example. The MSFWT labeling of the graph is stable when the seed (represented by $\circ$ ) of tree $A$ is moved within its core (cf. Fig. 3(a)(b): after cutting arc 2 , minimum weight is 4 ). It does nevertheless change when seed $A$ is moved out of its core, though still within its initial tree (cf. Fig. 3(c): after cutting arc 3, minimum weight is 3 ).

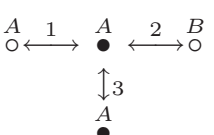

(a)

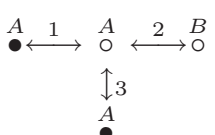

(b)

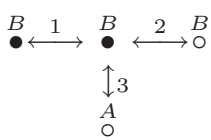

(c)

\section{Figure 3. Non-robustness of the MSF when} the seed $(\circ)$ is moved within a tree.

Many MSF-WTs are sometimes possible. In this case, we should consider a unified partition (TZ-MSF-WT) built from these solutions by tie-zone transform. However, the catchment basins, complement of the tie-zone, do neither constitute a robustness region for the seed choice. Figure 4(a) shows a counter-example where two MSFs of weight 6 can be built from a graph with two seeds $A$ and $B$. If seed $A$ is moved out of its core, though within its catchment basin (Fig. 4(b)), a third MSF of weight 6 is possible, which modifies the TZ-MSF.

(a)

(b)

\begin{tabular}{|c|c|c|c|c|c|c|c|c|c|c|}
\hline & - $\stackrel{2}{\longleftrightarrow}$ & 0 & $\stackrel{1}{\longrightarrow}$ & $\bullet$ & $\stackrel{2}{\longleftrightarrow}$ & • & $\stackrel{1}{\longleftrightarrow}$ & • & 2 & 0 \\
\hline $\operatorname{MSF} 1(=6)$ & $\mathrm{A} \longleftrightarrow$ & $\mathrm{A}$ & $\longleftrightarrow$ & A & $\longleftrightarrow$ & $\mathrm{A}$ & $\longleftarrow$ & A & & B \\
\hline MSF $2(=6)$ & $\mathrm{A} \longleftrightarrow$ & A & $\longleftrightarrow$ & A & & B & $\longleftarrow$ & B & $\longrightarrow$ & $\rightarrow \mathrm{B}$ \\
\hline TZ- & A & A & & A & & $\mathrm{TZ}$ & & $\mathrm{TZ}$ & & B \\
\hline & $0 \stackrel{2}{\longleftarrow}$ & $\bullet$ & $\stackrel{1}{\longleftarrow}$ & • & 2 & $\bullet$ & $\stackrel{1}{\longleftarrow}$ & $\bullet$ & 2 & 0 \\
\hline MSF & $\mathrm{A} \longleftrightarrow$ & A & $\longleftrightarrow$ & A & $\longleftrightarrow$ & A & $\longleftrightarrow$ & A & & B \\
\hline MSF $2(=6)$ & $\mathrm{A} \longleftrightarrow$ & $\mathrm{A}$ & $\longleftrightarrow$ & A & & B & $\longleftrightarrow$ & $\mathrm{B}$ & $\longleftrightarrow$ & $\rightarrow \mathrm{B}$ \\
\hline MSF $3(=6)$ & A & B & $\longleftrightarrow$ & B & $\longleftrightarrow$ & B & $\longleftrightarrow$ & B & $\longleftrightarrow$ & $\rightarrow \mathrm{B}$ \\
\hline TZ-MSF & A & $\mathrm{TZ}$ & & $\mathrm{TZ}$ & & $\mathrm{TZ}$ & & $\mathrm{TZ}$ & & B \\
\hline
\end{tabular}

Figure 4. Non-robustness of the set of MSFs when the seed $(\circ)$ is moved within a tree.

Therefore, we demonstrate that the robustness regions are contained in the trees and correspond to the cores characterized by Eqs. 4 and 5. Indeed, the result of the segmentation by TZ-MSF-WT is stable when we modify the position of a seed within the core of its tree: It will not change the labeling associated to the set of MSFs. To prove it, we propose the following theorem. 

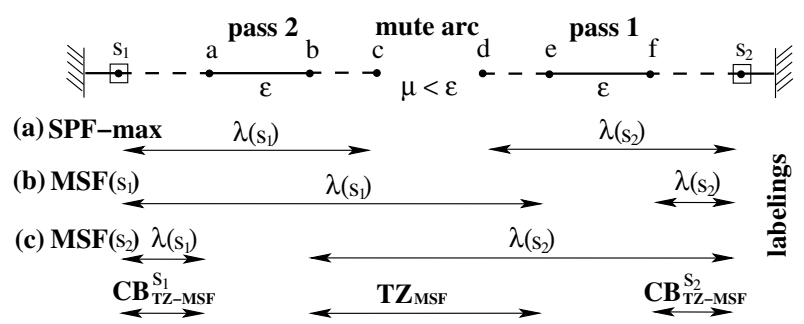

Figure 5. Extreme pass and mute arcs in cycle $\sigma^{c d}$.

Theorem 1 Let $(V, A, w)$ be a weighted graph, $w$ a nonnegative weight function. Let $S \subseteq V$ be a set of seeds. The tie-zone transform of the minimum spanning forests is equal to the tie-zone transform of the shortest path forests using the max-arc path cost function $f_{\max }$ :

$$
\begin{aligned}
T Z_{M S F} & =T Z_{S P F-\max } \\
C B_{T Z-M S F}(s) & =C B_{T Z-S P F-\max }(s), \forall s \in S
\end{aligned}
$$

Consequently, if the seeds $s$ are chosen randomly in their respective cores $K_{s}$, the TZ-IFT-WT (TZ-SPF-max) is unchanged, and so is the TZ-MSF-WT. Therefore the cores for the TZ-MSF-WT method are characterized by the same Eqs. 4 and 5.

Demonstration of Theorem 1: If $p \in T Z_{M S F}$, there are at least two MSFs that give different labeling for $p$. According to Proposition 1, these MSFs are also IFT-WT. As there are at least two IFT-WTs that give different labelings for $p$, $p \in T Z_{I F T-W T}$. If $p \in C B_{T Z-I F T-W T}(s)$, all the IFTWTs give the same labeling for $p$. So, all the MSFs, which are necessarily IFT-WTs, give the same labeling for $p$. So, $p \in C B_{T Z-M S F}$. So we have: $T Z_{M S F} \subseteq T Z_{I F T-W T}$ and $C B_{T Z-I F T-W T}(s) \subseteq C B_{T Z-M S F}(s), \forall s \in S$.

Now we demonstrate that the IFT-WTs (or shortest path forests with $f_{\max }$, SPF-max) that are not MSFs do not affect the tie zone of the MSFs. Note that the MSF of a weighted graph $(V, A, w)$ can be viewed as a minimum spanning tree of the same graph to which a ficticious root node $z$ was added. Arcs of weight -1 link $z$ to each seed. The minimum spanning tree satisfies the following theorem:

Theorem 2 (Minimum spanning tree [7]) $(V, T)$ is a tree of minimum weight for graph $(V, A, w)$ if and only if for every arc $u \in A \backslash T$ the cycle $\sigma^{u}$ (such that $\sigma^{u} \subset T \cup\{u\}$ ) satisfies: $w(u) \geq w(v), \forall v \in \sigma^{u}(v \neq u)$.

Let $F=\left(V, A^{\prime}\right)$ be an SPF-max of graph $(V, A, w)$ that is not MSF (cf. Fig. 5(a)). $F$ is not MSF. So, considering the derived tree $(V \cup\{z\}, T)$ and Theorem 2, there exists at least one arc $\langle c, d\rangle \in A \backslash A^{\prime}$ such that: if this arc is added, a cycle $\sigma^{c d} \subset T \cup\{\langle c, d\rangle\}$ is created and $w(c, d)=\mu<$ $\epsilon, \epsilon=\max _{\forall u \in \sigma^{c d}}\{w(u)\}$. Suppose the cycle includes two seeds $s_{1}$ and $s_{2}$ and $\langle a, b\rangle$ is or one of the arcs of maximum weight in the cycle: $w(a, b)=\epsilon$. Without loss of generality, say that it is the nearest arc from $s_{1}$ with such a weight, so it is between $s_{1}$ and $\langle c, d\rangle$ in $F$ (cf. Fig. 5). We call it the extreme pass arc for $s_{2}$. The path cost $C[c]$ at $c$ in $F$ is: $C[c]=f_{\max }\left(\pi\left(s_{1}, c\right)\right)=\epsilon$.

As $F$ is an SPF-max, $f_{\max }\left(\pi\left(s_{1}, c\right)\right) \leq f_{\max }\left(\pi\left(s_{2}, c\right)\right)$. So, $\epsilon \leq \max \left\{f_{\max }\left(\pi\left(s_{2}, d\right)\right), \mu\right\}$. If $f_{\max }\left(\pi\left(s_{2}, d\right)\right) \leq \mu$, the previous inequality would lead to $\epsilon \leq \mu<\epsilon$, which is impossible. Consequently, $\epsilon \leq f_{\max }\left(\pi\left(s_{2}, d\right)\right)$. As $\epsilon$ is the maximum weight in the cycle $\sigma^{\text {cd }}$, the path cost $C[d]$ at $d$ in $F$ is necessarily: $C[d]=f_{\max }\left(\pi\left(s_{2}, d\right)\right)=\epsilon$. Hence, there exists at least one arc $\langle e, f\rangle$ between $d$ and $s_{2}$ in $F$, such that: $w(e, f)=\epsilon$. Suppose that, if there are many of such arcs, it is the nearest one from $s_{2}$. We call it the extreme pass arc for $s_{1}$.

Now, let us add arc $\langle c, d\rangle$ and remove $\langle e, f\rangle$, the extreme pass arc for $s_{1}$, so as not to introduce a cycle in the forest. Therefore, labels of nodes $d$ to $e$ change: $\lambda\left(s_{1}\right)$ instead of $\lambda\left(s_{2}\right)$ (cf. Fig. 5(b)). The total weight of the forest decreases by $\epsilon-\mu$. But path costs at nodes $d$ to $e$ remain equal to $\epsilon$. So, the forest remains a SPF-max.

Then, we search for other arcs $\langle c, d\rangle$ and $\langle e, f\rangle$ and make substitution again to iteratively decrease the weight of the forest until a minimum weight. When no more such arcs are found, the SPF-max will have become an MSF (cf. Theorem 2). Observe that the cycle $\sigma^{c d}$ created may include only one seed or even no seed. In this case, we make the same substitution to decrease the weight of the forest, but this does not affect the labeling.

In the case of including two seeds, suppose that at each substitution, advantage is given to $s_{1}$. So, the $M S F\left(s_{1}\right)$ will show the maximum extension of $s_{1}$ ) (cf. Fig. 5(b)). Now, the same series of substitutions is made but giving advantage to $s_{2}, s_{3}, \ldots \in S$ to get $M S F\left(s_{2}\right), M S F\left(s_{3}\right), \ldots$ (cf. Fig. 5(c)). Clearly, any labeling of node in an SPFmax has already been proposed by some of the $M S F\left(s_{i}\right)$ labelings. Thus, the labelings given by the SPF-max that are not MSFs do not affect the tie zone transform of the MSFs. Therefore, the tie-zone transforms of SPF-max (IFT-WT) and MSF are identical.

\section{Minimal sets of seeds}

We now look at the inverse problem of segmentation by watershed: the minimal seed set (MSS) problem. Given a partition obtained by WT, it consists in finding the minimal sets of seeds that reproduce the same segmentation by WT. When first addressed in ref. [9], it was developed only in the case of segmentation by MSF-WT where the graph nodes were primitive catchment basins (i.e., resulting from 
a classical watershed from regional minima). The problem is relevant and particularly interesting in the applications of video segmentation. From an initial segmentation made by the user, it is possible to minimize the set of seeds to recover this input segmentation (first frame). Then, we only have to propagate this minimal set of seeds to segment the next frames, estimating the new position of few seeds. Thus, it decreases the dimension of the estimation problem. Here, we extend the problem of MSS to any given partition where each segmented region corresponds to a connected region with differentiated label. Thus, the input partition can vary at the pixel level precision, instead of the primitive basin level. We propose an algorithm to compute the non-redundant receptive regions in each of which at least one pixel seed has to be selected to recover the input partition. We also show the relation between the particular regions of the MSS and WT seed robustness frameworks: core, catchment basin, tie-zone, non-redundant receptive region, redundant receptive region, and dead zone.

\subsection{Definitions}

For completeness of the article, we first recall the main definitions of ref. [9].

Let $L$ be the aimed labeling representing a segmentation (or partition) of the image-graph $(V, A, w)$ : each segment or part of the partition is connected and represented by a different label $\lambda \in \Lambda$. The frontier nodes $F(\lambda)$ of a segment $\lambda$ are those nodes neighbors of other segment(s):

$$
F(\lambda)=\{p \in V, \exists q \in N(p), L[q] \neq L[p]=\lambda\}
$$

The strength $\mathcal{S}(p)$ of a frontier node $p$ is the minimum cost of the paths that connect it to other segments, i.e. the minimum weight of the arcs linking to neighbors of different label:

$$
\mathcal{S}(p)=\min \{w(p, q), q \in N(p), L[q] \neq L[p]\}
$$

The receptive region $R R(p)$ associated to frontier node $p$ is the set of nodes of the same segment which are linked to $p$ by a path whose cost is lower than the strength of $p$ :

$$
\begin{array}{r}
R R(p)=\{q \in V, L[q]=L[p], \exists \pi(p, q), \\
\left.f_{\max }(\pi(p, q))<\mathcal{S}(p)\right\}
\end{array}
$$

Property 1 If the receptive regions of two frontier nodes of a segment intersect, that of greater strength contains the other: $p, q \in F(\lambda), R R(p) \cap R R(q) \neq \emptyset, \mathcal{S}(p) \geq \mathcal{S}(q) \Rightarrow$ $R R(p) \supseteq R R(q)$.

A receptive region is redundant if it strictly contains another receptive region:

$$
R R(p) \text { redundant } \Leftrightarrow \exists q, R R(q) \subset R R(p)
$$

otherwise, it is said non-redundant. The dead zone $D Z(\lambda)$ of a segment $\lambda$ consists of the nodes of the segment which are in none of its receptive region:

$$
D Z(\lambda)=\{p \in V, L[p]=\lambda, p \notin R R(q), \forall q \in F(\lambda)\}
$$

We build a minimal seed set by choosing one seed node in each non-redundant receptive region of each segment, assigned by the respective label. Labeling $L$ is thereby recovered by WT from any minimal seed set. Additional seeds in either dead zone, redundant or non-redundant regions have no effect on segmentation, as long as they are assigned to their respective label.

\subsection{Relationship with cores and tie-zone}

Looking at these definitions, we see that the minimum seed set determination must be related to the problem of finding cores. Indeed, they are very close problems. Both aim to a goal partition stability. The great difference between the core robustness and the minimum seed set problems is that the former deals with the stability of the overall tree spans (not the trees) while the latter focus on the stability of the label span. In general, many seeds (therefore, trees) can share the same label of the target partition. In sum, they are similar problems but their level of application is different. Moreover, their inputs are different: for given tree seeds, the cores are determined as the regions where they can move while either these seeds and the receptive regions where they can move are unknown.

In fact, we demonstrate that the core problem is implicit in the MSS problem: Once the MSS problem has been solved, one has to pick a seed in each non-redundant receptive region (NRRR). At that moment, the core problem is automatically solved for all seeds: the core corresponds to the respective NRRR.

Demonstration: Let $R R(p), p \in F(\lambda)$ be a NRRR and $p$ the seed we picked to constitute part of a minimum seed set $S$. From Definition 9, there does not exist $q$ such that $R R(q) \subset R R(p)$, i.e. if $\forall q \in F(\lambda), R R(p) \cap R R(q) \neq \emptyset$, $\Rightarrow R R(p) \subseteq R R(q)$. And according to Property $1, \mathcal{S}(p) \leq$ $\mathcal{S}(q)^{1}$ for such frontier nodes $q$. Using the pass-value definition (cf. Section 3.1) and Eq. 8, we have:

$$
R R(p)=\{v \in V, L[v]=L[p], \epsilon(p, v)<\mathcal{S}(p)\} .
$$

As $\mathcal{S}(p)$ is the minimum weight of arcs linking to a node of different label (cf. Eq. 7), the pass-value between seed $p$ and any other seed of different label $s$ is:

$$
\epsilon(p, s) \geq \mathcal{S}(p), \forall s \in S, L[s] \neq L[p] .
$$

Consider now the seeds $t \in S$ that have the same label $L[t]=L[p]$ but do not belong to the same NRRR:

\footnotetext{
${ }^{1}$ We will take advantage of this result in our algorithm (Section 4.3).
} 
$t \notin R R(p)$. Hence, $\forall \pi(p, t), f_{\max }(\pi(p, t)) \geq \mathcal{S}(p)$ (cf. Eq. 8) and so:

$$
\epsilon(p, t) \geq \mathcal{S}(p), \forall t \in S, L[t]=L[p]
$$

It results from Eqs. 11, 12 and 13 that: $R R(p)=$ $\{v \in V, L[v]=L[p], \epsilon(p, v)<\epsilon(p)\}$, which is equivalent to core $K_{p}$ (cf. Eq. 5).

If more seeds than the minimum number are chosen, the cores, which make the spans of each individual tree stable, do no longer correspond to the NRRRs and must be determined separately. The labeling, yet, will be stable. If just a seed per NRRR is picked and assigned by its respective target label, the set of NRRRs referring to a label can be viewed as a distributed minimum core relative to the label.

Observe that there is no tie-zone in the MSS because the target partition has only labeled regions without tiezone. Indeed, allowing multiple NRRRs with the same label avoids the creation of TZ. As the TZ transform applied on any minimal set of seeds results in the target partition without tie-zone, the catchment basins (that correspond to tree spans) are the same for MSF-WT, IFT-WT, TZ-MSF-WT and TZ-IFT-WT (due to Theorem 1).

\subsection{Algorithm}

The brute force algorithm for computing the MSS would consist in computing the receptive regions of all frontier nodes, and their possible intersections to conserve only the non-redundant ones. Thus, the image pixels might be processed many times. The proposed algorithm uses two optimizations so as to process each pixel at most only once, and less when there are redundant regions or dead zone:

1. During the detection of receptive regions $R(p)$, nodes whose path cost is greater than or equal to strength $S(p)$ are not processed.

2. The receptive regions $R(p)$ are detected in increasing order of strength $S(p)$.

The input graph must have symmetric arcs, the nodes may represent either pixels or primitive catchment basins. In lines 1 and 2, frontier pixels are detected and strengths computed, according to Eqs. 6 and 7. Then, for each label $\lambda$, the receptive regions $R(p)$ are detected in increasing order of strength $S(p)$ (lines 3-5).

The DetectRR function (lines 7-18) has a main loop (lines 9-17) that uses an ordered queue $Q$ to propagate frontier node index $p$ in the nodes $u$ whose path cost is less than the strength of $p$. Note that index is propagated to the only neighbors with same label $L[u]=L[p]$ and which have not already been definitively processed (line 13). Minimum path cost is computed and updated in lines 14-17 and node $u$ is inserted in $Q$. The first optimization is made in line
15. Propagation is interrupted for the nodes with path cost greater than the strength $s$ of $p$. These nodes are neither inserted nor removed from $Q$. So, not all the nodes should be processed, as it is the case of the nodes in the dead zones for example.

Each node is removed at most once from queue $Q$ (lines 10-12). Map $P$, initialized with value NIL, associates to the processed (removed) node $v$ the index of the propagated frontier pixels $p$ if $v \in R R(p)$ (line 12). But before that, we check if this node $v$ has already been associated to another $R R\left(p^{\prime}\right)$ (line 11). If so, the propagation loop is interrupted (second optimization). It means that $R R\left(p^{\prime}\right)$ intersects $R R(p)$, and as $S\left(p^{\prime}\right) \leq S(p)$ (increasing processing order), $R R(p)$ is redundant. It is not worth computing it. In this case, $p$ is not a frontier node that propagated its index into an non-redundant receptive region. So, it does not belong to the set $N R I$ of indices of frontier nodes resulting in a non-redundant receptive region (line 18).

In a final step (line 6), we copy the index of the respective frontier node located in $P[p]$ into map $N R[p]$, when $p$ belongs to an NRRR. Indeed, map $P$ contains some frontier node indices whose propagation was interrupted.

\section{Pseudocode 1: Non-redundant receptive region (NRRR) detection}

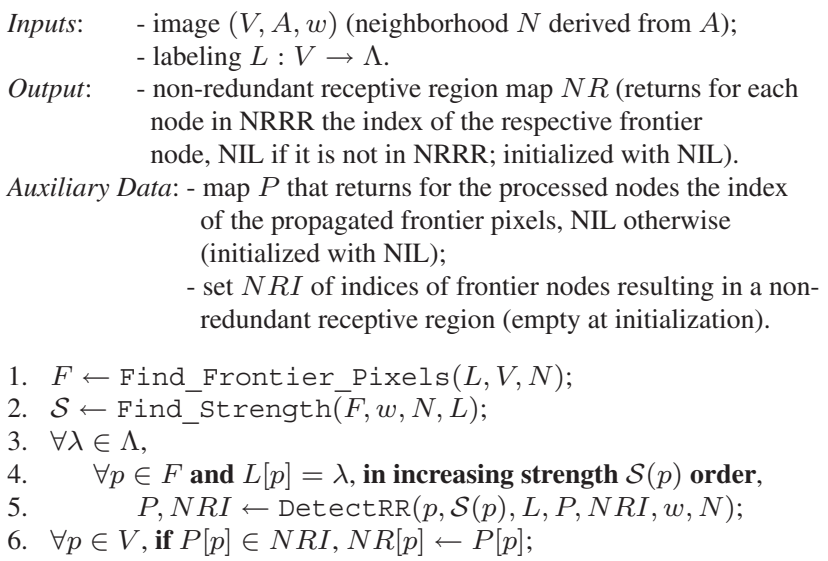

18. if nobreak $=$ TRUE, $N R I \leftarrow N R I \cup\{p\}$; 


\section{Conclusion}

This paper analyzed the problem of robustness relative to input seeds for three segmentation approaches: the iterative relative fuzzy object extraction, the watershed transforms by image foresting transform and by minimum spanning forest. It dealt with the characterization of the seed cores on the one hand, and with the minimal sets of seed, on the other hand.

New theoretical results which were not intuitive nor trivial were demonstrated. They allow to link around the robustness topic problems and approaches that were known as a-priori different, like shortest-path forest, minimum spanning forest, fuzzy connectedness and watershed. So we showed that the set of shortest-path forests with max-arc path cost gives the same segmentation as the set of minimum spanning forests, and, therefore, that they have the same core characterization as in the fuzzy connectedness framework. The equivalence between cores of a minimal seed set and non-redundant receptive regions was proved too. Moreover, a new algorithm was proposed for the computation of minimal seed sets at pixel level.

We hope these theoretical results will contribute to the understanding and evaluation of segmentation robustness. Robustness measures based on core area and distribution could be useful to prevent not very repeatable segmentation results, or evaluate the effect of a prefiltering stage. The visualization of minimum seed sets, redundant receptive regions and dead zones could help the user with interactive segmentation routines. For example, he/she could select only the satisfactory regions of a first segmentation, and compute the minimal seed sets, which could guide him/her for a refined segmentation by showing that it is or not worth picking seeds with some label in some region.

\section{Acknowledgments}

This work is supported by CAPES.

\section{References}

[1] R. Audigier and R. Lotufo. Duality between the watershed by image foresting transform and the fuzzy connectedness segmentation approaches. In XIX Brazilian Symp. on Computer Graph. and Image Proc. (SIBGRAPI'06), pages 5360, Manaus, Brazil, Oct. 2006. IEEE Press.

[2] R. Audigier and R. Lotufo. Watershed by image foresting transform, tie-zone, and theoretical relationships with other watershed definitions. In 8th International Symposium on Mathematical Morphology (ISMM'07), Rio de Janeiro, Brazil, Oct. 2007.

[3] R. Audigier, R. Lotufo, and M. Couprie. The tie-zone watershed: Definition, algorithm and applications. In Proceedings of IEEE International Conference on Image Processing
(ICIP'05), volume 2, pages 654-657, Genova, Italy, Sept. 2005.

[4] S. Beucher and C. Lantuéjoul. Use of watersheds in contour detection. In International Workshop on Image Processing, Real-Time Edge and Motion Detection/Estimation, Rennes, France, 1979.

[5] S. Beucher and F. Meyer. The Morphological Approach to Segmentation: The Watershed Transform. In E. R. Dougherty, editor, Mathematical Morphology in Image Processing, chapter 12, pages 433-481. Marcel Dekker, Inc., New York (NY), USA, 1993.

[6] A. Falcão, J. Stolfi, and R. Lotufo. The image foresting transform: Theory, algorithms, and applications. IEEE Trans. on Pattern Analysis and Machine Intelligence, 26(1):19-29, Jan. 2004.

[7] M. Gondran and M. Minoux. Trees and arborescences. In Graphs and algorithms, chapter 4, pages 129-159. John Wiley \& Sons Ltd., 1984.

[8] G. T. Herman and B. M. Carvalho. Multiseeded segmentation using fuzzy connectedness. IEEE Trans. Pattern Anal. Mach. Intell., 23(5):460-474, 2001.

[9] R. Lotufo and W. Silva. Minimal set of markers for the watershed transform. In 6th International Symposium on Mathematical Morphology (ISMM), page 359368, Sydney, Australia, 2002. CSIRO publications.

[10] F. Meyer. Minimum spanning forests for morphological segmentation. In J. Serra and P. Soille, editors, Mathematical morphology and its applications to image processing, pages 77-84. Kluwer Academic Publishers, 1994.

[11] F. Meyer. Topographic distance and watershed lines. Signal Processing, (38):113-125, 1994.

[12] J. Udupa, P. Saha, and R. Lotufo. Relative fuzzy connectedness and object definition: Theory, algorithms, and applications in image segmentation. IEEE Tr. on PAMI, 24(11):1485-1500, November 2002.

[13] J. Udupa and S. Samarasekera. Fuzzy connectedness and object definition: Theory, algorithms, and applications in image segmentation. Graph. Models Image Process., 58(3):246-261, May 1996. 